\title{
Synthesis and Characterization of Biodegradable Synthetic Thickener from Anionic Triglyceride Polylactic Acid
}

\author{
Meram S. Abdelrahman ${ }^{1}$, Sahar H. Nassar ${ }^{1, *}$, Hamada Mashaly ${ }^{1}$, Safia Mahmoud ${ }^{1}$, Dalia Maamoun² \\ ${ }^{1}$ Dyeing, Printing and Auxiliaries Department, Textile Research Division, National Research Centre, Cairo 12622, Egypt \\ ${ }^{2}$ Faculty of Applied Arts, Helwan University, Cairo, Egypt \\ *Corresponding author: sh.wahab@nrc.sci.eg
}

\begin{abstract}
In this research work, we report the synthesis of novel biodegradable synthetic thickener able to provide good quality prints with reduced wastewater pollution. Biodegradable triglyceride polylactic acid (TGPLA) star polymer hydrogel was prepared via one-pot reaction by direct polycondensation of lactic acid and glycerol. The chemical structure of the produced polymer was determined By Fourier transform infrared spectroscopy (FT-IR), nuclear magnetic resonance spectroscopy $\left({ }^{1} \mathrm{H}-\mathrm{NMR}\right)$, and gel permeation chromatography (GPC). The rheological properties and biodegradation of the novel thickening agent were investigated. The prints quality of the prepared thickening hydrogels increased with increasing lactic acid content, while the apparent viscosity and biodegradation rate increased. Technical evaluation of TGPLA as environmentally friendly thickening agent in printing of different fabrics was studied. In addition, the chemical oxygen demand (COD) and biological oxygen demand (BOD) were described. The use of such thickening composite in printing such variety of fabrics with pigment and different types of dyes including reactive, acid, metal complex, basic, and disperse dyes was described. It was established that the TGPLA composite paste is characterize by a non-Newtonian pseudoplastic performance, and their apparent viscosity increases upon neutralization. Printing pastes are very stable against storing compared to mypro-gum as natural thickener. Printing pastes thickened with TGPLA containing a ratio of $98 \%$ of lactic acid and $2 \%$ of glycerol; display similar $K / S$ values and colorfastness properties compared to those prints from commercial synthetic thickeners.
\end{abstract}

Keywords: lactic acid, triglyceride star polymer, synthetic thickener, textile printing, biodegradability

Cite This Article: Meram S. Abdelrahman, Sahar H. Nassar, Hamada Mashaly, Safia Mahmoud, and Dalia Maamoun, "Synthesis and Characterization of Biodegradable Synthetic Thickener from Anionic Triglyceride Polylactic Acid.” Applied Ecology and Environmental Sciences, vol. 6, no. 2 (2018): 35-47. doi: 10.12691/aees-6-2-1.

\section{Introduction}

The use of biodegradable additives able to provide good quality prints with reduced wastewater pollution attracted recently significant attention [1,2]. In textile printing, synthetic thickeners are used as thickening agent to avoid improper fabric handle. Polyacrylic acids synthetic thickeners are extensively used in textile printing [3,4,5]. However, polyacrylates are prepared from acrylic acid which causes severe irritation to skin and respiratory tract, while high exposure could result in pulmonary edema. Eye contact with acrylic acid also causes severe and irreversible injuries. The oral lethal dose $\mathrm{LD}_{50}$ in rat is estimated as $340 \mathrm{mg} / \mathrm{kg}$ [6-11]. In addition, polyacrylic acid as synthetic thickener usually displays poor outline sharpness of prints and is not biodegradable, leading to higher wastewater pollution [4]. Furthermore, the uses of natural thickeners in textile printing cause fabric stiffness and exhibit no stability for storing [12,13,14,15]. Due to petroleum shortage, production of polymers from renewable resources has become more significant. There has been growing attention in utilization poly(lactic acid) in a variety of applications because of its natural basis and high biodegradability. However, its implementation within textile industries has been restricted to lower temperature wet and dry processing, due to its comparatively lower glass transition temperature and melting point which results in low thermal stability and low impact resistance [16-21]. On the other hand, triglyceride oils have been utilized in the synthesis of a variety of polymers. Triglyceride oils can be considered as one of the most significant resources for biopolymers. Oil-based biopolymers possess various advantages compared to polymers from petroleum-based monomers which are not biodegradable and, in many cases, more expensive than the biodegradable oil-based biopolymers [22,23,24].

The utilization of biodegradable thickening agents presents an environmentally friendly printing practice with reduced pollution in effluent. Herein, we report the first eco-friendly TGPLA-based synthetic thickener for use in textile printing to minimize water pollution while maintaining good quality prints. Synthetic thickeners with anionic charges show great potential. We present the synthesis of novel anionic charged triglyceride polylactic 
acid synthetic thickener for textile printing on a variety of fabrics using different types of dyes. The synthesis involves polymerization reaction of lactic acid with glycerol at different ratios. The polymerization reaction gives rise to triglyceride polylactic acid of different ratios. These polymeric products will be referred to as thickening composites. Factors affecting the preparation of those composite thickeners along with their overall color fastness properties of the prints are investigated.

\section{Experimental Section}

\subsection{Apparatus and Methods}

The COD values were measured by KIT COD/1500 by POT Dichromate APP Nanocor 300D and heating block Nanocolor R-8. The TOC concentration was obtained by subtracting the inorganic carbon concentration from the total carbon concentration using Shimadzu TOC-5000 Analyzer, Japan. FTIR spectra were recorded by using an FTIR spectrophotometer (Nexus 670, Nicolet, USA) in the region of $4000-400 \mathrm{~cm}^{-1}$ with spectra resolution of $4.0 \mathrm{~cm}^{-1}$. NMR spectra were recorded using a BRUKER AVANCE 400 spectrometer at $400 \mathrm{MHz}$ employing deuterated DMSO- $\mathrm{d}_{6}$; chemical shifts are reported in ppm relative to tetramethylsilane internal standard at $295^{\circ} \mathrm{K}$. The color characteristics of the printed fabrics were determined on a Texflash ACS/Datacolor with a Spectraflash 600 spectrophotometer. The fastness properties were determined at Textile Research Division, National Research Centre, Egypt. The pH measurements were recorded with AD-11 ADWA digital $\mathrm{pH}$ meter. Molecular weight of TGPLA was measured by gel permeation chromatography (GPC, Hewlett Packard 1100 Series) using polystyrene as the standard and $\mathrm{CHCl}_{3}$ as the mobile phase.

All experimental results were collected at room temperature unless stated otherwise. Solvents used in this study were obtained from Fluka and Aldrich and were employed without further purification. Lactic acid (90\%) was purchased from Aladdin Industrial Corporation. All reactions were monitored by thin layer chromatography (TLC) using Merck aluminum plates pre-coated with silica gel $\mathrm{PF}_{254}$; $20 \times 20 \mathrm{~cm}, 0.25 \mathrm{~mm}$, and detected by visualization of the plate under UV lamp (254 or $365 \mathrm{~nm}$ ).

\subsection{Materials and Supplies}

\subsubsection{Commercial Thickening Agents}

Sodium alginate. Sodium alginate of medium viscosity type manufactured by CeCa Kolloid Chemie, Paris France, was Kindly Supplied under the commercial name Ceca alginate HV/KL 600.

Myprogum. NP-16(Meyhall), which is a non-ionic thickening agent based on modified plant seed gum, was used at a concentration of $8 \%$.

Synthetic thickener. Daico Thick 1600, synthetic thickener was kindly supplied by Daico Company, Cairo, Egypt.

\subsubsection{Fabrics}

$100 \%$ cotton, $100 \%$ polyester, $100 \%$ wool, $100 \%$ acrylic, $100 \%$ nylon, cotton/polyester (50:50), and wool/polyester
(55:45) fabrics were supplied by El-Mahalla El-Kobra Company, El-Mahalla, Egypt. The fabric were scoured in aqueous solution having a liquor ratio of 1:50 and containing $2 \mathrm{~g} \mathrm{~L} \mathrm{~L}^{-1}$ of non-ionic detergent solution (Hostapal, Clariant, Swiss) and $2 \mathrm{~g} \mathrm{~L}^{-1}$ of sodium carbonate at $60^{\circ} \mathrm{C}$ for 30 minutes to get rid of waxes and impurities, followed by rinsing in cold water, and finally dried at room temperature.

\subsubsection{Dyes and Pigment}

All colorants were kindly supplied by El-Mahalla El-Kobra Company, El-Mahalla, Egypt; including Realan Rot RC Granulat (Reactive Dye), Isolan Orange K-RLS 150\% (Acid Dye), Suncron Blue FBL D.BL56 (Disperse Dye), Maxilon Golden Yellow GL 400\% (Basic Dye), Pigment Orange 34, and Isolan Bordo S-BLN (Metal Complex Dye).

\subsubsection{Binder}

Printofix Binder MTB was kindly supplied by Clariant.

\subsubsection{Other chemicals}

Lactic acid, glycerol, sodium carbonate, sodium bicarbonate, ammonium persulfate, hydroquinone, and urea were of laboratory grade chemicals.

\subsection{Synthesis of Triglyceride Polylactic Acid (TGPLA) Polymer}

Triglyceride polylactic acid polymers with various molar ratios of glycerol and lactic acid were synthesized via one-step polycondensation procedure as shown in Scheme 1. The typical synthesis is proceeded as follows (for ratio: $50 \% \mathrm{LA}$ to $50 \% \mathrm{GL}$ ): A mixture of lactic acid (18 g, $20 \mathrm{mmol})$ and glycerol (17.2 g, $20 \mathrm{mmol})$ was stirred in a $500 \mathrm{~mL}$ conical flask, while raising the temperature to $90^{\circ} \mathrm{C}$. 2-3 drops of concentrated sulfuric acid were added. The reaction was then heated at $100^{\circ} \mathrm{C}$ for additional 20-30 minutes. After cooling, concentrated solution of sodium hydroxide was gradually added to neutralize the reaction mixture. The mixture was then subjected to water evaporation using rotary evaporator to obtain the pure solid content. The polymer composite can also be separated by addition of ethanol to afford a wet gel that can be filtered, dried and milled.

\subsection{Preparation of Printing Pastes}

\subsubsection{Acid and Metal Complex Dyes}

The printing pastes were prepared according to the following recipe:

Dye 30

Urea 50

Ethylene glycol 50

Thickener $\quad \mathrm{Y}$

Water $\quad \mathrm{X}$

Ammonium Sulphate(1:2) 60

Ludigol 15 
The amount of thickener used was either Myprogum $80 \mathrm{~g} / \mathrm{kg}$ printing paste or triglyceride polylactic acid $80 \mathrm{~g} / \mathrm{kg}$.

\subsubsection{Disperse Dye}

The printing pastes were prepared according to the following recipe:-

$\begin{array}{ll}\text { Dye } & 40 \\ \text { Water } & \text { X } \\ \text { Sodium dihydrogen Phosphate } & 20 \\ \text { Ludigol } & 5 \\ \text { Thickener } & \text { Y }\end{array}$

40

$\mathrm{X}$

20

Y

1000

The amount of thickener used was either Synthetic Thickener $30 \mathrm{~g} / \mathrm{kg}$ printing paste or triglyceride polylactic acid $80 \mathrm{~g} / \mathrm{kg}$.

\subsubsection{Reactive Dye}

$\begin{array}{ll}\text { Dye } & 30 \\ \text { Urea } & 50 \\ \text { Ethylene glycol } & 50 \\ \text { Thickener } & \mathrm{Y} \\ \text { Water } & \mathrm{X} \\ \text { Formic acid } & 10 \\ \text { Ludigol } & 15 \\ & ------ \\ & 1000\end{array}$

The amount of thickener used was either Sodium Alginate $40 \mathrm{~g} / \mathrm{kg}$ printing paste or triglyceride polylactic acid $80 \mathrm{~g} / \mathrm{kg}$.

\subsubsection{Basic Dye}

$\begin{array}{ll}\text { Dye } & 30 \\ \text { Acetic Acid30\% } & 30 \\ \text { Ethylene glycol } & 3 \\ \text { Thickener } & \mathrm{Y} \\ \text { Hot Water } & \mathrm{X} \\ \text { Citric acid } & 5 \\ & ------ \\ & 1000\end{array}$

The amount of thickener used was either Sodium Alginate $40 \mathrm{~g} / \mathrm{kg}$ printing paste or triglyceride polylactic acid $80 \mathrm{~g} / \mathrm{kg}$.

\subsubsection{Pigment}

$\begin{array}{ll}\text { Pigment } & 40 \\ \text { Thickener } & \mathrm{Y} \\ \text { Binder } & 40 \\ \text { Diamonum phosphate } & 10 \\ \text { Water } & \mathrm{X} \\ & ------ \\ & 1000\end{array}$

The amount of thickener used was either Synthetic Thickener $30 \mathrm{~g} / \mathrm{kg}$ printing paste or triglyceride polylactic acid $80 \mathrm{~g} / \mathrm{kg}$.

\subsection{Printing Technique}

All the aforementioned printing pastes were applied to cotton, polyester, wool, acrylic, nylon, cotton/polyester, and wool/polyester fabrics using screen printing technique. Fixation of the printed garments was performed either by steaming for printed fabrics by acid, metal complex, basic, or reactive dye (at $100-103^{\circ} \mathrm{C}$ for 30 minutes) or thermofixation for printed fabrics by pigment and disperse dye (at $150^{\circ} \mathrm{C}$ for 5 minutes for pigment and $180^{\circ} \mathrm{C}$ for 5 minutes for disperse dye) in an automatic thermostatic oven (Wenner Mathis Co., Switzerland).

\subsection{Washing Process}

Washing of printed fabrics with pigment, metal complex, basic, and reactive dyes:

Rinsing with cold water, hot water and soaping of the prints with $2 \mathrm{~g} / \mathrm{l}$ nonionic detergent at $60^{\circ} \mathrm{C}$ for 30 minutes.

Washing of printed fabrics with acid dye:

Washing of printed goods was carried out as follows: Rinsing with cold water- Soaping at $30-40^{\circ} \mathrm{C}$ in $2 \mathrm{~g} / \mathrm{l}$ soap solution- Rinsing with cold water

Reduction clear and washing of printed fabrics with disperse dye:

- Rinsing in cold water

- Soaping 60 at $^{\circ} \mathrm{C}$ with $2 \mathrm{~g} / \mathrm{l}$ Hostapal CV ( non ionic detergent) for 20 minutes.

- Reduction clear with 2g/l hydrosulphite, 2g/l sodium hydroxide (32.5\%), 2g/l Hostapl CV (non ionic detergent) at $40-70^{\circ} \mathrm{C}$.

- Rinsing at $60-70^{\circ} \mathrm{C}$.

- Cold rinsing.

Finally, the samples were dried and assessed for $K / S$ and fastness properties.

\subsection{Determination of Rheological Properties}

The Rheological properties of the printing pastes are expressed as the apparent viscosity at different rates of shear; were measured using Brookfield Model DV-III Programmable Rheometer.

\subsection{Colorfastness Measurements}

The color strength, expressed as K/S and the overall fastness properties (washing, crocking, perspiration, and light), were assessed according to the standard techniques $[25,26,27,28,29]$.

\subsection{Biodegradability}

Biodegradability tests were carried out according to OECD Guidelines for Testing Chemicals employing procedure 301D (closed bottle test) [30]. The biological oxygen demand $\left(\mathrm{BOD}_{10}\right)$ value is usually expressed in milligrams of oxygen consumed per liter of sample during 10 days of incubation at $20^{\circ} \mathrm{C}$ and is often used as a surrogate of the degree of organic pollution of water. BOD can be used as a gauge of the effectiveness of wastewater treatment plants. 


\section{Results and Discussion}

\subsection{Synthesis and Structural Characterization}

Novel biodegradable triglyceride polylactic acid TGPLA cross-linked polymer hydrgel was prepared via one-pot reaction by thermal polycondensation of lactic acid and glycerol at different ratios in presence of sulfuric acid as a catalyst. The chemical structure of the TGPLA polymer was demonstrated by FT-IR, ${ }^{1} \mathrm{H}-\mathrm{NMR}$, and GPC. The prints quality of the gels increased with increasing LA contents, while the biodegradation rate increased. LA is polymerized via polycondensation in presence of sulfuric acid catalyst, and the polymerization mechanism is shown in Scheme 1. As was expected, the properties of the obtained polymer composites depend on the concentration ratio of monomers, i.e. glycerol and lactic acid. Hence, ten different triglyceride/polylactic acid composite samples were synthesized by changing the amounts of both lactic acid and glycerol monomers as formerly stated in the experimental part. Strong broad peak at $3426 \mathrm{~cm}^{-1}$ is assigned to the carboxylic group. Two IR peaks (Supplementary Information) at 2988 and $2939 \mathrm{~cm}^{-1}$ are assigned to the absorption of the aliphatic groups including $\mathrm{CH}, \mathrm{CH}_{2}$, and $\mathrm{CH}_{3}$. Peaks at 1736 and $1642 \mathrm{~cm}^{-}$ ${ }^{1}$ are assigned to the absorption of carbonyl ester and carbonyl amide groups, respectively, in TGPLA98. The ${ }^{1} \mathrm{H}-\mathrm{NMR}$ chemical shifts (Supplementary Information) at $\delta 4.32$ and $1.12 \mathrm{ppm}$ are attributed to the protons of the $\mathrm{C}(\mathrm{O}) \mathrm{CH}$ and $\mathrm{CHCH}_{3}$ groups in the lactic acid units. Peaks visible at $\delta 1.27$ and 3.59 are assigned to the central glycerol $\mathrm{CH}$ and the $\mathrm{CH}_{2}$ of the glycerol units respectively. These results signify the successful synthesis of the TGPLA polymer. The molar ratios of LA to GL were 98/2, 90/10, 80/20, 70/30, 60/40, 50/50, 40/60, 30/70, $20 / 80$ and 10/90. Thus, the TGPLA samples with different compositions were abbreviated depending on the ration of lactic acid monomer as TGPLA98, TGPLA90, TGPLA80, TGPLA70, TGPLA60, TGPLA50, TGPLA40, TGPLA30, TGPLA20 and TGPLA10, respectively. These results meant that the molecular weights of the TGPLA polymers could be controlled by adjusting the molar ratio of lactic acid to glycerol monomers. The molecular weight of TGPLA increased as longer polymerisation time proceeded. The longer polymerization time leads to high molecular weight solid state polymer. The most suitable molecular weight which was around 36,000 was obtained at 30 minutes. The TGPLA composite is miscible with water and can be separated as a soft gel by addition of ethanol as it is not soluble in water/ethanol mixture, so it can be isolated from the mixture, air-dried and finally milled. The weight average molecular weights $(M \mathrm{w})$ of TGPLA98, from gel permeation chromatography (GPC) measurements, were controlled from 19,000 to 48,000 by changing the polycondensation reaction proceeding time as listed in Table 1.

\subsection{Rheological Properties of Printing Pastes}

The screening started with the investigation of ten different concentration ratios of LA and GL. The rheological properties after neutralization of the ten composite samples were investigated before and after storing for 1 and 3 days as represented in Table S2 Table S4 respectively. It is clear that irrespective of the concentration of lactic acid monomer and/or the time elapsed before commence measuring, the relation between the shearing stress and rate of shear is not liner indicating that, these pastes are not Newtonian.

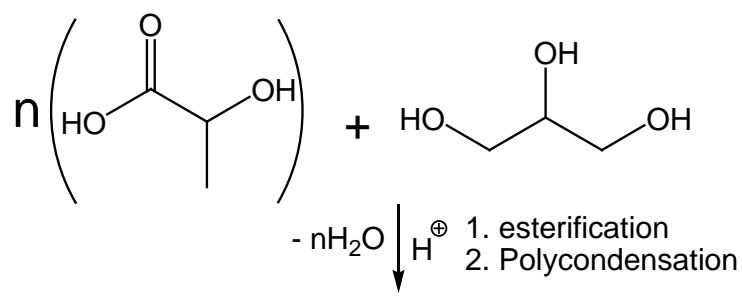

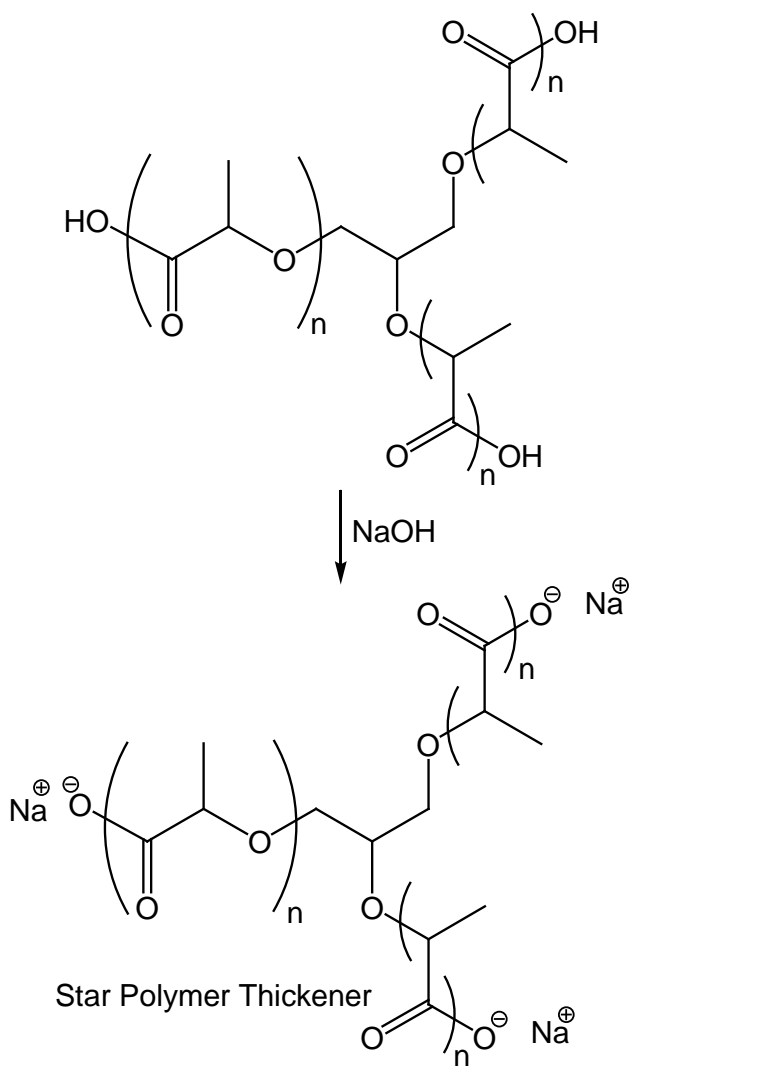

Scheme 1. Synthesis of triglyceride polylactic acid polymer

Table 1. Molecular weights of TGPLA98 copolymer upon increasing reaction time

\begin{tabular}{|l|c|}
\hline Processing Time (min.) & $M_{\mathrm{W}}\left(\mathrm{x} 10^{4}\right)$ \\
\hline 20 & 3.6 \\
40 & 7.7 \\
60 & 10.1 \\
80 & 14.0 \\
100 & 16.9 \\
\hline
\end{tabular}

Rate of shear was ranging between 22.8 and 101.4. For example, if the rate of shear is held constant at $21.3 \mathrm{sec}^{-1}$, the apparent viscosity increases from 283 to 574, 1520, to 3931Centipoise by increasing the concentration of TGPLA from $8,11,14$, to 17 respectively. The influence of glycerol monomer on paste rheology is investigated. It is also clear that as the concentration of lactic acid monomer increases, the apparent viscosity increases 
regularly irrespective of the rate of shear. A marked reduction in the apparent viscosity curves was detected upon increasing the concentration of glycerol monomer which can be explained in terms of the increased crosslinking between glycerol and lactic acid. As the concentration of the latter raises up, the length of PLA side chains increases leading to the increment in the molecular weight, and consequently an increment in the resistance of polymer flow, i.e., an increment in apparent viscosity. On the other hand, at a constant concentration, the apparent viscosity decreases by increasing the rate of shear indicates that, these paste exhibit squaring pseudo plastic behavior. The decrease in the apparent viscosity by increasing the rate of shear means that, if the viscosity (resistance to flow) of a solution is recorded using a large applied fabric (shearing stress) which causes a high velocity of flow (shear rate), the viscosity is less than that of the same solution determined with slower rate of flow (Table 2; Figure 1 and Figure 2). In pseudo plastic solutions, no time dependence effect is observed. Furthermore, it is clear that, storing of the aforementioned pastes for 1 or 3 days at room temperature has no effect on the rheological properties of the printing paste, where it remains not Newtonian pseudo plastic. It is also obvious that the TGPLA thickener is stable for storing since the variation in the values of viscosity after storing is very marginal.

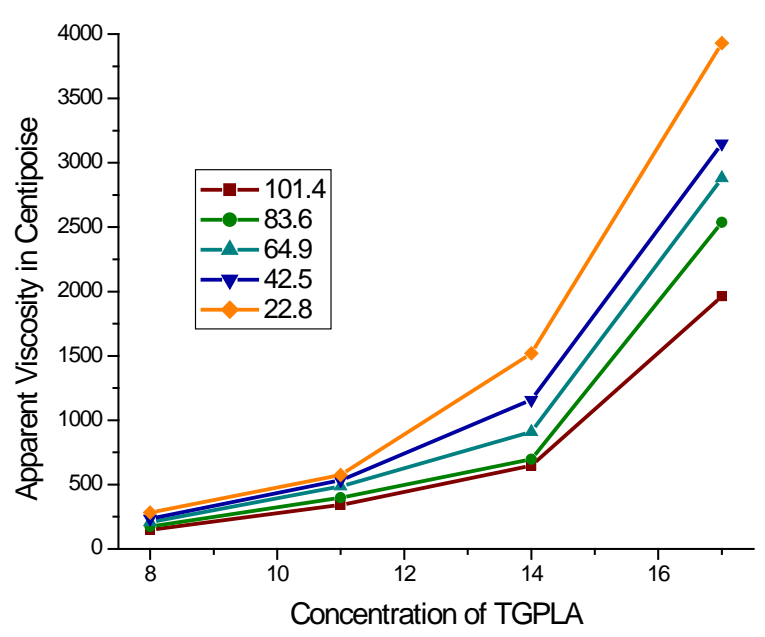

Figure 1. Effect of polymer concentration on the apparent viscosity at different rates of shear

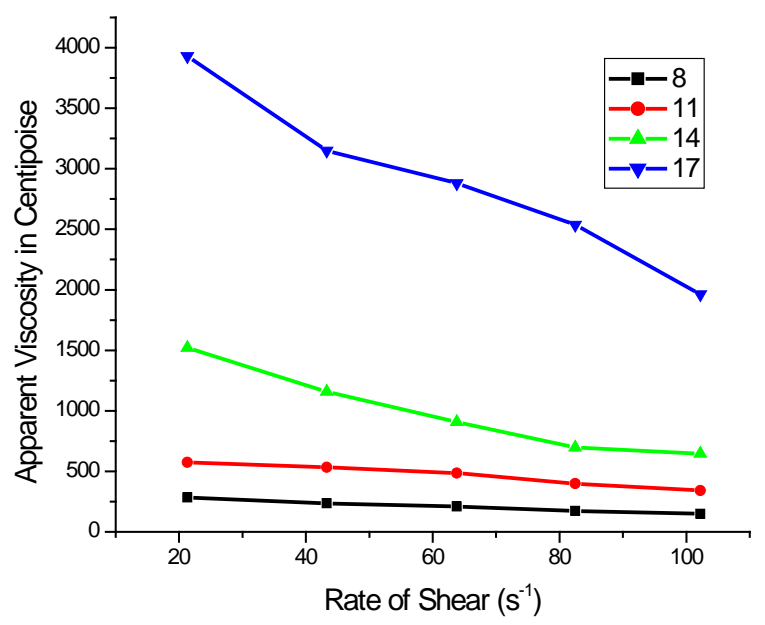

Figure 2. Rheogram display dependence of the apparent viscosity on the rate of shear at diferent concentrations of TGPLA
Table 2. Effect of TGPLA polymer concentration in water on the thickening process and apparent viscosity at different rate of shear

\begin{tabular}{|c|c|c|c|c|c|}
\hline \multirow{2}{*}{$\begin{array}{c}\text { Concentration } \\
\text { of TGPLA }\end{array}$} & \multicolumn{5}{|c|}{ Apparent Viscosity in Centipoise at Rate of Shear } \\
\cline { 2 - 6 } & 22.8 & 42.5 & 64.9 & 83.6 & 101.4 \\
\hline $8 \%$ & 283 & 236 & 209 & 174 & 149 \\
\hline $11 \%$ & 574 & 533 & 486 & 398 & 341 \\
\hline $14 \%$ & 1520 & 1158 & 908 & 697 & 647 \\
\hline $17 \%$ & 3931 & 3150 & 2880 & 2537 & 1962 \\
\hline
\end{tabular}

It was observed that neutralization of the TGPLA polymer composite directly after preparation has an effect on the rheological properties. The increasing in viscosity upon neutralization was surprising and may be due to the following reasons: (1) an increase in the polymer molecular weight by replacing hydrogen (atomic wt; 1) of the lactic acid carboxylic group with sodium (atomic wt; 23) to afford the sodium salt of the composite, (2) an increase in the adhesive force, or (3) an increase in the degree of formation of $\mathrm{COO}^{-} \mathrm{Na}^{-}$functional group more than that of the $\mathrm{COOH}$ carboxylic group, and consequently, the repulsion forces among the polymer chains increases. As the extent of repulsion increases, the orientation of the composite polymer molecules along the rotation axis becomes harder, and consequently, the resistance to flow increases.

\subsection{Printing Results}

To investigate the suitability of the neutralized composite (TGPLA) as thickener in printing, three different concentration ratios of LA and GL were selected (LA/GL; 10/90, 50/50, and 98/2). At similar conditions, these composites were printed on different fabrics (cotton, polyester, wool, acrylic, nylon, cotton/polyester and wool/polyester) with different colorant (Reactive, Acid, Basic, disperse, metal and pigment. For comparison, we employed additional printing pastes thickened with sodium alginate in case of reactive and Basic dye, or commercial synthetic thickener (Daico thick) in case of pigment and disperse dye or mypro gum in case of acid and metal dyes. After printing, the colored samples were air-dried and fixed by means of streaming or thermofixation as previously mentioned in the experimental section. The samples were then washed thoroughly, directed and their color strength $(K / S)$ as well as their overall fastness properties was measured (Table S5 Table S17). The use of high concentration of the glycerol monomer caused a lower paste add-on and therefore effected a small reduction in color strength but contributed to a soft fabric handle. The best fabric handle achieved using TGPLA98 and was comparable to that of prints produced with myprogum, sodium alginate or synthetic thickener. The best printing results and a very soft fabric handle were obtained when TGPLA98 at a concentration of up to $8 \%$ was used. The K/S of prints produced using TGPLA98 as a thickener is higher than that produced when commercial thickener was used. In addition, the prints are soft and acquire no harsh feeling. Current results suggest that the TGPLA are soluble in water and can be removed easily during washing, thereby not causing any adverse consequences on handling of the printed textile substrates. Other natural thickeners pastes are usually not appropriate for storing and are highly affected by 
fermentation; bad odor evolves upon storing for more than 24 hours and they lose most of the resistance to flow (i.e. viscosity). TGPLA can be easily washed off during the washing process. The TGPLA composites carry carboxyl groups which cannot intereact with a reactive colorant and play a main role in repelling the similarly charged reactive dye ions and therefore, a high color yield is obtained.

\subsection{Biodegradation}

The biodegradation property is very important for biobased polymers. An eco-friendly printing practice requires biodegradable printing pastes because chemicals and auxiliaries used in the printing process will be washed out following printing and fixation. A complete study on biodegradation was carried out for TGPLA composite polymers at different concentration ratios of both lactic acid and glycerol monomers. Table 3 displays the biodegradation magnitudes of these pastes. The biodegradation examinations have shown that the printing pastes made of TGPLA are more favorable than those pastes made of sodium alginate or commercial synthetic thickeners (all samples at the same concentration of $1 \mathrm{~g} \mathrm{l}^{-1}$ ). This means that the use of TGPLA as thickening agent in printing pastes can lead to a significant wastewater de-pollution. Due to the high ratio of lactic acid, TGPLA98 displayed the highest biodegradable polymer with suitable apparent viscosity for printing a variety of fabrics using a variety of colorants.

Table 3. Biodegradation analysis of commercial synthetic thickener, sodium alginate, and TGPLA polymer in aqueous medium (Conc. $1 \mathrm{~g} \mathrm{l}^{-1}$ )

\begin{tabular}{|c|c|c|c|}
\hline $\begin{array}{c}\text { Percent of } \\
\text { LA: } \mathrm{GL}\end{array}$ & $\begin{array}{c}{ }^{*} \mathrm{COD} \\
\left(\mathrm{mg} \mathrm{O}_{2} \mathrm{~L}^{-1}\right)\end{array}$ & $\begin{array}{c}{ }^{* *} \mathrm{BOD}_{10} \\
\left(\mathrm{mg} \mathrm{O}_{2} \mathrm{~L}^{-1}\right)\end{array}$ & $\begin{array}{c}{ }^{* * *} \mathrm{BOD}_{10} / \mathrm{COD} \\
(\mathrm{x} \mathrm{100 \%})\end{array}$ \\
\hline $10: 90 \%$ & 222 & 62 & 27.9 \\
\hline $20: 80 \%$ & 228 & 63 & 27.6 \\
\hline $30: 70 \%$ & 231 & 61 & 26.4 \\
\hline $40: 60 \%$ & 233 & 65 & 27.9 \\
\hline $50: 50 \%$ & 238 & 68 & 29.6 \\
\hline $60: 40 \%$ & 244 & 71 & 29.2 \\
\hline $70: 30 \%$ & 253 & 74 & 29.5 \\
\hline $80: 20 \%$ & 261 & 77 & 30.2 \\
\hline $90: 10 \%$ & 265 & 80 & 31.7 \\
\hline $98: 02 \%$ & 274 & 87 & \\
\hline
\end{tabular}

* Chemical Oxygen Demand; ** Biological Oxygen Demand; *** Biological Oxygen Demand in 10 days.

\section{Conclusion}

Novel aliphatic random TGPLA polymer was successfully prepared from lactic acid and glycerol monomers via polycondensation procedure using sulfuric acid as a catalyst. The produced composite is evaluated as thickening agent in textile printing. The molecular weight and composition of the TGPLA polymers could be adapted by altering the molar ratio of lactic acid and glycerol. The thickener pastes are distinguished by non-Newtonian pseudoplastic properties, and their viscosity rises upon increasing the concentration ratio of lactic acid. The composite can be diluted by water and its apparent viscosity increases upon neutralization. Printing pastes of TGPLA composite thickener are very stable to storing. The K/S values and color fastness properties of prints obtained with the TGPLA thickener are equivalent to those produced by using commercial thickeners. Additionally, the use of such biodegradable thickener results in reduction of wastewater pollution. The produced polymer thickener composed of lactic acid and glycerol monomers can afford the customer with advantages in terms of environmental biocompatibility and soft handle.

\section{Acknowledgements}

We gratefully acknowledge National Research Centre, Cairo, Egypt; for the support of this work.

\section{References}

[1] Mohammad Shahid, and Faqeer Mohammad. "Perspectives for natural product based agents derived from industrial plants in textile applications-a review." Journal of cleaner production 57 (2013): 2-18.

[2] Reinhold Schneider, and Sonja Šostar-Turk. "Good quality printing with reactive dyes using guar gum and biodegradable additives." Dyes and pigments 57, no. 1 (2003): 7-14.

[3] A. A. Hebeish, A. A. Ragheb, S. H. Nassar, E. E. Allam, and J. I. El Thalouth. "Polymerization products of acrylic acid with Gleditsia triacanthos gum as thickeners for reactive printing." Journal of applied polymer science 101, no. 2 (2006): 931-943.

[4] M. H. Abo-Shosha, M. R. El-Zairy, and N. A. Ibrahim. "Preparation and rheology of new synthetic thickeners based on polyacrylic acid." Dyes and pigments 24, no. 4 (1994): 249-257.

[5] Laurence G. Dammann, "Polyacrylate thickening agents." U.S. Patent 4,338,239, issued July 6, 1982.

[6] R. R. Miller, J. A. Ayres, G. C. Jersey, and M. J. McKenna. "Inhalation toxicity of acrylic acid." Fundamental and Applied Toxicology 1, no. 3 (1981): 271-277.

[7] H. Greim, J. Ahlers, R. Bias, B. Broecker, H. Hollander, H-P. Gelbke, S. Jacobi et al. "Assessment of structurally related chemicals: toxicity and ecotoxicity of acrylic acid and acrylic acid alkyl esters (acrylates), methacrylic acid and methacrylic acid alkyl esters (methacrylates)." Chemosphere 31, no. 2 (1995): 2637-2659.

[8] Göksel N. Demirer, and Richard E. Speece. "Toxicity of acrylic acid to acetate-enriched methanosarcina cultures." Journal of Environmental Engineering 124, no. 4 (1998): 345-352.

[9] H-J. Klimisch, and J. Hellwig. "The prenatal inhalation toxicity of acrylic acid in rats." Fundamental and applied toxicology 16, no. 4 (1991): 656-666.

[10] T. L. Neeper-Bradley, E. H. Fowler, I. M. Pritts, and T. R. Tyler. "Developmental toxicity study of inhaled acrylic acid in New Zealand white rabbits." Food and chemical toxicology 35, no. 9 (1997): 869-880.

[11] John Autian, "Structure-toxicity relationships of acrylic monomers." Environmental health perspectives 11 (1975): 141.

[12] Sandeep Bains, and Manisha Sethi. "Cassia Gum an Environmentfriendly Alternative Thickener in Printing with Natural Dyes." International Journal of Bio-Resource \& Stress Management 8, no. 1 (2017).

[13] S. Sostar, and R. Schneider. "Guar gum as an environmentfriendly alternative thickener in printing with reactive dyes." Dyes and pigments 39, no. 4 (1998): 211-221.

[14] Lili Wang, Rumeng Li, Jianzhong Shao, and Zhao Wang. "Rheological behaviors of carboxymethyl tamarind gum as thickener on georgette printing with disperse dyes." Journal of Applied Polymer Science 134, no. 26 (2017). 
[15] A. A. Ragheb, Nassar, S. H., El-Thalouth, I. A., Ibrahim, M. A., \& Shahin, A. A. (2012). Preparation, characterization and technological evaluation of CMC derived from rice-straw as thickening agents in discharge, discharge-resist and burn-out printing. Carbohydrate polymers, 89(4), 1044-1049.

[16] Yan Guo, Chuanfu Wu, Qunhui Wang, Min Yang, Qiqi Huang, Markus Magep, and Tianlong Zheng. "Wastewater-nitrogen removal using polylactic acid." Frontiers of Environmental Science \& Engineering 10, no. 4 (2016): 1-10.

[17] Kate Parker, Jean-Philippe Garancher, Samir Shah, and Alan Fernyhough. "Expanded polylactic acid-an eco-friendly alternative to polystyrene foam." Journal of Cellular Plastics 47, no. 3 (2011): 233-243.

[18] Tanveer Hussain, Muhammad Tausif, and Munir Ashraf. "A review of progress in the dyeing of eco-friendly aliphatic polyester-based polylactic acid fabrics." Journal of Cleaner Production 108 (2015): 476-483.

[19] Joseph K. Muiruri, Songlin Liu, Wern Sze Teo, Junhua Kong, and Chaobin He. "Highly Biodegradable and Tough Polylactic AcidCellulose Nanocrystal Composite." ACS Sustainable Chemistry \& Engineering 5, no. 5 (2017): 3929-3937.

[20] Fatma Masmoudi, Atef Bessadok, Mohamed Dammak, Mohamed Jaziri, and Emna Ammar. "Biodegradable packaging materials conception based on starch and polylactic acid (PLA) reinforced with cellulose." Environmental Science and Pollution Research 23, no. 20 (2016): 20904-20914.

[21] Hossein Izadi-Vasafi, Gity Mir, Mohamad Sadeghi, Amir Babaei, and Faezeh Ghayoumi. "A novel biodegradable polyurethane based on hydroxylated polylactic acid and tung oil mixtures. I. Synthesis, physicochemical and biodegradability characterization." Fibers and Polymers 17, no. 3 (2016): 311.
[22] F. Seniha Güner, Yusuf Yağc1, and A. Tuncer Erciyes. "Polymers from triglyceride oils." Progress in Polymer Science 31, no. 7 (2006): 633-670.

[23] Zahra Jeirani, Badrul Mohamed Jan, Brahim Si Ali, Ishenny Mohd Noor, Chun Hwa See, and Wasan Saphanuchart. "The effect of polymer on triglyceride microemulsion formulation for tertiary oil recovery." Industrial \& Engineering Chemistry Research 52, no. 44 (2013): 15589-15593.

[24] S. Begila David, K. Sathiyalekshmi, and G. Allen Gnana Raj. "Studies on acrylated epoxydised triglyceride resin-co-butyl methacrylate towards the development of biodegradable pressure sensitive adhesives." Journal of Materials Science: Materials in Medicine 20, no. 1 (2009): 61.

[25] ISO 105-C02:1989 Textiles: Tests for color fastness, Part C02: Color fastness to washing: Test 2 (Basel: ISO, 1989).

[26] ISO 105-X12:1987 Textiles: Tests for color fastness, Part X12: Color fastness to rubbing (Basel: ISO, 1987).

[27] ISO 105-E04:1989 Textiles: Tests for color fastness, Part E04: Color fastness to perspiration (Basel: ISO, 1989).

[28] ISO 105-B02:1988 Textiles: Tests for color fastness, Part B02: Color fastness to artificial light: Xenon arc fading lamp test (Basel: ISO, 1988).

[29] W. Baumann, N. Kleinemeier, R. Brossman, H. P. Oesch, M. Krayer, B. T. Groebel, A. T. Leaver, J. Soc. Dyers Colour. 1987, 103, 100-105.

[30] International Organization for Standardization. Standard ISO 10707. water quality "Evaluation in an aqueous medium of ultimate aerobic bio degradability of organic compounds". Method by analysis of biochemical oxygen demand (closed bottle test), Geneva, 1994.

\section{Electronic Supplementary Information}

\section{Synthesis, characterization and properties of biodegradable synthetic thickener from anionic triglyceride polylactic acid}

Table S1. Different concentration ratios of used monomers

\begin{tabular}{|c|c|c|}
\hline Percent of Lactic acid to Glycerol & Weight of Lactic Acid & Weight of Glycerol \\
\hline $10: 90 \%$ & $3.6 \mathrm{~g}$ & $29.12 \mathrm{~g}$ \\
\hline $20: 80 \%$ & $7.2 \mathrm{~g}$ & $25.76 \mathrm{~g}$ \\
\hline $30: 70 \%$ & $10.8 \mathrm{~g}$ & $22.08 \mathrm{~g}$ \\
\hline $40: 60 \%$ & $14.4 \mathrm{~g}$ & $18.4 \mathrm{~g}$ \\
\hline $50: 50 \%$ & $18.0 \mathrm{~g}$ & $14.72 \mathrm{~g}$ \\
\hline $60: 40 \%$ & $21.6 \mathrm{~g}$ & $11.04 \mathrm{~g}$ \\
\hline $70: 30 \%$ & $25.2 \mathrm{~g}$ & $7.36 \mathrm{~g}$ \\
\hline $80: 20 \%$ & $28.8 \mathrm{~g}$ & $3.68 \mathrm{~g}$ \\
\hline $90: 10 \%$ & $32.4 \mathrm{~g}$ & $0.736 \mathrm{~g}$ \\
\hline $98: 02 \%$ & $35.28 \mathrm{~g}$ & \\
\hline
\end{tabular}

Table S2. Effect of concentration of lactic acid on the apparent viscosity of the obtained TGPLA composite freshly prepared

\begin{tabular}{|c|c|c|c|c|c|}
\hline \multirow{2}{*}{ Percent of Lactic/Glycerol } & \multicolumn{5}{|c|}{ Apparent viscosity in Centipoise at Rate of shear $\left(\mathrm{s}^{-1}\right)$ before storing (Fresh paste) } \\
\hline & 17.3 & 18.4 & 20.7 & 21.4 & 24.8 \\
\hline $10: 90 \%$ & 680 & 667 & 564 & 554 & 551 \\
\hline $20: 80 \%$ & 694 & 684 & 681 & 679 & 675 \\
\hline $30: 70 \%$ & 1145 & 1103 & 993 & 989 & 971 \\
\hline $40: 60 \%$ & 2580 & 2206 & 2135 & 2134 & 2131 \\
\hline $50: 50 \%$ & 2650 & 2413 & 2363 & 2253 & 2211 \\
\hline $60: 40 \%$ & 2831 & 2826 & 2369 & 2261 & 2257 \\
\hline $70: 30 \%$ & 2893 & 2887 & 2708 & 2673 & 2647 \\
\hline $80: 20 \%$ & 2984 & 2903 & 2867 & 2742 & 2663 \\
\hline $90: 10 \%$ & 3011 & 3001 & 2985 & 2973 & 2964 \\
\hline $98: 02 \%$ & 3925 & 3814 & 3766 & 3618 & 3549 \\
\hline
\end{tabular}


Table S3. Effect of storing for 1 day on the paste thickened with the TGPLA composite has been prepared using different concentration of Lactic Acid

\begin{tabular}{|c|c|c|c|c|c|}
\hline \multirow{2}{*}{ Percent of Lactic/Glycerol } & \multicolumn{5}{|c|}{ Apparent viscosity in Centipoise at Rate of shear $\left(\mathrm{s}^{-1}\right)$ after one day storing } \\
\hline & 17.3 & 18.4 & 20.7 & 21.4 & 24.8 \\
\hline $10: 90 \%$ & 679 & 567 & 549 & 535 & 498 \\
\hline $20: 80 \%$ & 688 & 674 & 667 & 604 & 598 \\
\hline $30: 70 \%$ & 1133 & 989 & 946 & 931 & 911 \\
\hline $40: 60 \%$ & 2489 & 1105 & 1103 & 1029 & 1009 \\
\hline $50: 50 \%$ & 2560 & 1342 & 1235 & 1233 & 1122 \\
\hline $60: 40 \%$ & 2793 & 1452 & 1392 & 1290 & 1236 \\
\hline $70: 30 \%$ & 2811 & 1703 & 1621 & 1549 & 1456 \\
\hline $80: 20 \%$ & 2894 & 1989 & 1975 & 1963 & 1835 \\
\hline $90: 10 \%$ & 2898 & 2113 & 2105 & 2019 & 1958 \\
\hline $98: 02 \%$ & 3997 & 3852 & 3742 & 3694 & 3589 \\
\hline
\end{tabular}

Table S4. Effect of storing 3 days on the paste thickened with the TGPLA composite has been prepared using different concentration of Lactic Acid

\begin{tabular}{|c|c|c|c|c|c|}
\hline \multirow{2}{*}{ Percent of Lactic/Glycerol } & \multicolumn{5}{|c|}{ Apparent viscosity in Centipoise at Rate of shear $\left(\mathrm{s}^{-1}\right)$ after three days storing } \\
\hline & 17.3 & 18.4 & 20.7 & 21.4 & 24.8 \\
\hline $10: 90 \%$ & 653 & 552 & 545 & 528 & 519 \\
\hline $20: 80 \%$ & 675 & 673 & 669 & 534 & 524 \\
\hline $30: 70 \%$ & 1124 & 1113 & 1110 & 1089 & 953 \\
\hline $40: 60 \%$ & 2439 & 1304 & 1204 & 1097 & 981 \\
\hline $50: 50 \%$ & 2532 & 2401 & 2330 & 1287 & 1102 \\
\hline $60: 40 \%$ & 2622 & 2503 & 2412 & 1331 & 1198 \\
\hline $70: 30 \%$ & 2731 & 2513 & 2501 & 1890 & 1345 \\
\hline $80: 20 \%$ & 2744 & 2613 & 2609 & 1934 & 1689 \\
\hline $90: 10 \%$ & 3801 & 3704 & 3676 & 3576 & 3485 \\
\hline $98: 02 \%$ & 7250 & 7000 & 6705 & 6511 & 6323 \\
\hline
\end{tabular}

Table S5. Comparison between $\mathrm{K} / \mathrm{S}$ and fastness properties of wool fabric sample printed with prepared different TGPLA composite thickeners along with those printed using Mypro-Gum when acid used as a dye

\begin{tabular}{|c|c|c|c|c|c|c|c|c|c|c|c|}
\hline \multirow{4}{*}{ Thickening agent } & \multirow{4}{*}{ Storing Time } & \multirow{4}{*}{$\mathrm{K} / \mathrm{S}$} & \multicolumn{8}{|c|}{ Fasten properties } & \multirow{4}{*}{ Light } \\
\hline & & & \multirow{2}{*}{\multicolumn{2}{|c|}{ Rubbing }} & \multirow{2}{*}{\multicolumn{2}{|c|}{ Washing }} & \multicolumn{4}{|c|}{ Perspiration } & \\
\hline & & & & & & & \multicolumn{2}{|c|}{ Acidic } & \multicolumn{2}{|c|}{ Alkaline } & \\
\hline & & & Dry & Wet & St. & Alt & St. & Alt. & St. & Alt. & \\
\hline Mypro Gum & $\mathrm{F}$ & 13.72 & $4-5$ & 4 & $4-5$ & $4-5$ & $4-5$ & $4-5$ & $4-5$ & $4-5$ & $6-7$ \\
\hline Mypro Gum & S & 12.01 & $4-5$ & 4 & $4-5$ & $4-5$ & $4-5$ & $4-5$ & $4-5$ & $4-5$ & $6-7$ \\
\hline I & $\mathrm{F}$ & 12.15 & $4-5$ & 4 & $4-5$ & $4-5$ & $4-5$ & $4-5$ & $4-5$ & $4-5$ & 6-7 \\
\hline I & S & 12.45 & $4-5$ & 4 & $4-5$ & $4-5$ & $4-5$ & $4-5$ & $4-5$ & $4-5$ & $6-7$ \\
\hline II & $\mathrm{F}$ & 8.66 & $4-5$ & 4 & $4-5$ & $4-5$ & $4-5$ & $4-5$ & $4-5$ & $4-5$ & $6-7$ \\
\hline II & S & 17.37 & $4-5$ & $4-5$ & $4-5$ & $4-5$ & $4-5$ & $4-5$ & $4-5$ & $4-5$ & $6-7$ \\
\hline III & $\mathrm{F}$ & 18.08 & $4-5$ & 4 & $4-5$ & $4-5$ & $4-5$ & $4-5$ & $4-5$ & $4-5$ & $6-7$ \\
\hline III & $S$ & 17.91 & $4-5$ & 4 & $4-5$ & $4-5$ & $4-5$ & $4-5$ & $4-5$ & $4-5$ & $6-7$ \\
\hline
\end{tabular}

I, II, and III are TGPLA synthetic thickeners prepared at 10, 50, and 98 \% of lactic acid, respectively. F: Freshly prepared, S: Stored for 3 dyes, St: Staining, Alt: Alteration 
Table S6. Comparison between $\mathrm{K} / \mathrm{S}$ and fastness properties of wool fabric sample printed with prepared different TGPLA composite thickeners along with those printed using Mypro-Gum when Metal used as a dye

\begin{tabular}{|c|c|c|c|c|c|c|c|c|c|c|c|}
\hline \multirow{4}{*}{$\begin{array}{c}\text { Thickening } \\
\text { agent }\end{array}$} & \multirow{4}{*}{$\begin{array}{l}\text { Storing } \\
\text { Time }\end{array}$} & \multirow{4}{*}{$\mathrm{K} / \mathrm{S}$} & \multicolumn{8}{|c|}{ Fasten properties } & \multirow{4}{*}{ Light } \\
\hline & & & \multirow{2}{*}{\multicolumn{2}{|c|}{ Rubbing }} & \multirow{2}{*}{\multicolumn{2}{|c|}{ Washing }} & \multicolumn{4}{|c|}{ Perspiration } & \\
\hline & & & & & & & \multicolumn{2}{|c|}{ Acidic } & \multicolumn{2}{|c|}{ Alkaline } & \\
\hline & & & Dry & Wet & St. & Alt & St. & Alt. & St. & Alt. & \\
\hline Mypro Gum & $\mathrm{F}$ & 6.02 & $4-5$ & 4 & $4-5$ & $4-5$ & $4-5$ & $4-5$ & $4-5$ & $4-5$ & $6-7$ \\
\hline Mypro Gum & $\mathrm{S}$ & 6.83 & $4-5$ & 4 & $4-5$ & $4-5$ & $4-5$ & $4-5$ & $4-5$ & $4-5$ & $6-7$ \\
\hline I & $\mathrm{F}$ & 10.35 & $4-5$ & 4 & $4-5$ & $4-5$ & $4-5$ & $4-5$ & $4-5$ & $4-5$ & $6-7$ \\
\hline I & S & 7.07 & $4-5$ & 4 & $4-5$ & $4-5$ & $4-5$ & $4-5$ & $4-5$ & $4-5$ & $6-7$ \\
\hline II & $\mathrm{F}$ & 6.35 & $4-5$ & 4 & $4-5$ & $4-5$ & $4-5$ & $4-5$ & $4-5$ & $4-5$ & $6-7$ \\
\hline II & S & 6.92 & $4-5$ & 4 & $4-5$ & $4-5$ & $4-5$ & $4-5$ & $4-5$ & $4-5$ & $6-7$ \\
\hline III & $\mathrm{F}$ & 8.94 & $4-5$ & 4 & $4-5$ & $4-5$ & $4-5$ & $4-5$ & $4-5$ & $4-5$ & $6-7$ \\
\hline III & S & 8.63 & $4-5$ & 4 & $4-5$ & $4-5$ & $4-5$ & $4-5$ & $4-5$ & $4-5$ & $6-7$ \\
\hline
\end{tabular}

I, II, and III are TGPLA synthetic thickeners prepared at 10, 50, and 98 \% of lactic acid, respectively. F: Freshly prepared, S: Stored for 3 dyes, St: Staining, Alt: Alteration.

Table S7. Comparison between K/S and fastness properties of polyester fabric sample printed with prepared different TGPLA composite thickeners along with those printed using commercial Synthetic thickener(Dicothick) when disperse used as a dye.

\begin{tabular}{|c|c|c|c|c|c|c|c|c|c|c|c|}
\hline \multirow{4}{*}{ Thickening agent } & \multirow{4}{*}{ Storing Time } & \multirow{4}{*}{$\mathrm{K} / \mathrm{S}$} & \multicolumn{8}{|c|}{ Fasten properties } & \multirow{4}{*}{ Light } \\
\hline & & & \multirow{2}{*}{\multicolumn{2}{|c|}{ Rubbing }} & \multirow{2}{*}{\multicolumn{2}{|c|}{ Washing }} & \multicolumn{4}{|c|}{ Perspiration } & \\
\hline & & & & & & & \multicolumn{2}{|c|}{ Acidic } & \multicolumn{2}{|c|}{ Alkaline } & \\
\hline & & & Dry & Wet & St. & Alt & St. & Alt. & St. & Alt. & \\
\hline Dico thick & $\mathrm{F}$ & 17.19 & $4-5$ & 4 & $4-5$ & $4-5$ & $4-5$ & $4-5$ & $4-5$ & $4-5$ & $6-7$ \\
\hline Dico thick & $S$ & 17.26 & $4-5$ & 4 & $4-5$ & $4-5$ & $4-5$ & $4-5$ & $4-5$ & $4-5$ & $6-7$ \\
\hline I & $\mathrm{F}$ & 17.67 & $4-5$ & 4 & $4-5$ & $4-5$ & $4-5$ & $4-5$ & $4-5$ & $4-5$ & $6-7$ \\
\hline I & $S$ & 16.47 & 4 & 4 & $4-5$ & $4-5$ & $4-5$ & $4-5$ & $4-5$ & $4-5$ & $6-7$ \\
\hline II & $F$ & 15.39 & $4-5$ & 4 & $4-5$ & $4-5$ & $4-5$ & $4-5$ & $4-5$ & $4-5$ & $6-7$ \\
\hline II & $\mathrm{s}$ & 16.02 & $4-5$ & 4 & $4-5$ & $4-5$ & $4-5$ & $4-5$ & $4-5$ & $4-5$ & $6-7$ \\
\hline III & $\mathrm{F}$ & 16.80 & $4-5$ & 4 & $4-5$ & $4-5$ & $4-5$ & $4-5$ & $4-5$ & $4-5$ & $6-7$ \\
\hline III & $S$ & 16.64 & $4-5$ & 4 & $4-5$ & $4-5$ & $4-5$ & $4-5$ & $4-5$ & $4-5$ & $6-7$ \\
\hline
\end{tabular}

I, II, and III are TGPLA synthetic thickeners prepared at 10, 50, and 98 \% of lactic acid, respectively. F: Freshly prepared, S: Stored for 3 dyes, St: Staining, Alt: Alteration.

Table S8. Comparison between K/S and fastness properties of acrylic fabric sample printed with prepared different TGPLA composite thickeners along with those printed using commercial Synthetic thickener (Dico thick) when disperse used as a dye

\begin{tabular}{|c|c|c|c|c|c|c|c|c|c|c|c|}
\hline \multirow{4}{*}{ Thickening agent } & \multirow{4}{*}{ Storing Time } & \multirow{4}{*}{$\mathrm{K} / \mathrm{S}$} & \multicolumn{8}{|c|}{ Fasten properties } & \multirow{4}{*}{ Light } \\
\hline & & & \multirow{2}{*}{\multicolumn{2}{|c|}{ Rubbing }} & \multirow{2}{*}{\multicolumn{2}{|c|}{ Washing }} & \multicolumn{4}{|c|}{ Perspiration } & \\
\hline & & & & & & & \multicolumn{2}{|c|}{ Acidic } & \multicolumn{2}{|c|}{ Alkaline } & \\
\hline & & & Dry & Wet & St. & Alt & St. & Alt. & St. & Alt. & \\
\hline Dico thick & $\mathrm{F}$ & 23.69 & $4-5$ & 4 & $4-5$ & $4-5$ & $4-5$ & $4-5$ & $4-5$ & $4-5$ & $6-7$ \\
\hline Dico thick & $S$ & 21.55 & $4-5$ & 4 & $4-5$ & $4-5$ & $4-5$ & $4-5$ & $4-5$ & $4-5$ & $6-7$ \\
\hline I & $\mathrm{F}$ & 22.66 & $4-5$ & $4-5$ & $4-5$ & $4-5$ & $4-5$ & $4-5$ & $4-5$ & $4-5$ & $6-7$ \\
\hline I & $S$ & 22.28 & $4-5$ & $4-5$ & 4 & 4 & $4-5$ & $4-5$ & $4-5$ & $4-5$ & $6-7$ \\
\hline II & $\mathrm{F}$ & 16.16 & $4-5$ & $4-5$ & $4-5$ & $4-5$ & $4-5$ & $4-5$ & $4-5$ & $4-5$ & $6-7$ \\
\hline II & $S$ & 17.33 & $4-5$ & $4-5$ & $4-5$ & $4-5$ & $4-5$ & $4-5$ & $4-5$ & $4-5$ & $6-7$ \\
\hline III & $\mathrm{F}$ & 22.33 & $4-5$ & $4-5$ & $4-5$ & $4-5$ & $4-5$ & $4-5$ & $4-5$ & $4-5$ & $6-7$ \\
\hline III & $S$ & 22.43 & $4-5$ & 4 & $4-5$ & $4-5$ & $4-5$ & $4-5$ & $4-5$ & $4-5$ & $6-7$ \\
\hline
\end{tabular}

I, II, and III are TGPLA synthetic thickeners prepared at 10, 50, and $98 \%$ of lactic acid, respectively. F: Freshly prepared, S: Stored for 3 dyes, St: Staining, Alt: Alteration. 
Table S9. Comparison between K/S and fastness properties of Nylon fabric sample printed with prepared different TGPLA composite thickeners along with those printed using commercial Synthetic thickener (Dico thick) when disperse used as a dye

\begin{tabular}{|c|c|c|c|c|c|c|c|c|c|c|c|}
\hline \multirow{4}{*}{ Thickening agent } & \multirow{4}{*}{ Storing Time } & \multirow{4}{*}{$\mathrm{K} / \mathrm{S}$} & \multicolumn{8}{|c|}{ Fasten properties } & \multirow{4}{*}{ Light } \\
\hline & & & \multirow{2}{*}{\multicolumn{2}{|c|}{ Rubbing }} & \multirow{2}{*}{\multicolumn{2}{|c|}{ Washing }} & \multicolumn{4}{|c|}{ Perspiration } & \\
\hline & & & & & & & \multicolumn{2}{|c|}{ Acidic } & \multicolumn{2}{|c|}{ Alkaline } & \\
\hline & & & Dry & Wet & St. & Alt & St. & Alt. & St. & Alt. & \\
\hline Dico thick & F & 22.09 & $4-5$ & 4 & $4-5$ & $4-5$ & $4-5$ & $4-5$ & $4-5$ & $4-5$ & $6-7$ \\
\hline Dico thick & S & 19.60 & $4-5$ & 4 & $4-5$ & $4-5$ & $4-5$ & $4-5$ & $4-5$ & $4-5$ & $6-7$ \\
\hline I & $\mathrm{F}$ & 17.84 & $4-5$ & 4 & $4-5$ & $4-5$ & $4-5$ & $4-5$ & $4-5$ & $4-5$ & $6-7$ \\
\hline I & S & 15.58 & $4-5$ & 4 & $4-5$ & $4-5$ & $4-5$ & $4-5$ & $4-5$ & $4-5$ & $6-7$ \\
\hline II & $\mathrm{F}$ & 16.52 & $4-5$ & 4 & $4-5$ & $4-5$ & $4-5$ & $4-5$ & $4-5$ & $4-5$ & $6-7$ \\
\hline II & $\mathrm{S}$ & 14.67 & $4-5$ & 4 & $4-5$ & $4-5$ & $4-5$ & $4-5$ & $4-5$ & $4-5$ & $6-7$ \\
\hline III & $\mathrm{F}$ & 21.53 & $4-5$ & 4 & $4-5$ & $4-5$ & $4-5$ & $4-5$ & $4-5$ & $4-5$ & $6-7$ \\
\hline III & $\mathrm{S}$ & 18.83 & $4-5$ & 4 & $4-5$ & $4-5$ & $4-5$ & $4-5$ & $4-5$ & $4-5$ & $6-7$ \\
\hline
\end{tabular}

I, II, and III are TGPLA synthetic thickeners prepared at 10, 50, and 98 \% of lactic acid, respectively. F: Freshly prepared, S: Stored for 3 dyes, St: Staining, Alt: Alteration

Table S10. Comparison between K/S and fastness properties of wool fabric sample printed with prepared different TGPLA composite thickeners along with those printed using Sodium alginate when Reactive used as a dye

\begin{tabular}{|c|c|c|c|c|c|c|c|c|c|c|c|}
\hline \multirow{4}{*}{ Thickening agent } & \multirow{4}{*}{ Storing Time } & \multirow{4}{*}{$\mathrm{K} / \mathrm{S}$} & \multicolumn{8}{|c|}{ Fasten properties } & \multirow{4}{*}{ Light } \\
\hline & & & \multirow{2}{*}{\multicolumn{2}{|c|}{ Rubbing }} & \multirow{2}{*}{\multicolumn{2}{|c|}{ Washing }} & \multicolumn{4}{|c|}{ Perspiration } & \\
\hline & & & & & & & \multicolumn{2}{|c|}{ Acidic } & \multicolumn{2}{|c|}{ Alkaline } & \\
\hline & & & Dry & Wet & St. & Alt & St. & Alt. & St. & Alt. & \\
\hline Sodium alginate & $\mathrm{F}$ & 7.59 & 4 & 4 & $3-4$ & 4 & $3-4$ & 4 & 4 & 4 & $6-7$ \\
\hline Sodium alginate & S & 7.37 & 4 & 4 & $3-4$ & 4 & 4 & 4 & 4 & 4 & $6-7$ \\
\hline I & $\mathrm{F}$ & 8.29 & $4-5$ & $4-5$ & $3-4$ & 4 & 4 & 4 & $3-4$ & 4 & $6-7$ \\
\hline I & S & 8.52 & $4-5$ & 4 & $3-4$ & 4 & 4 & 4 & 4 & 4 & $6-7$ \\
\hline II & $\mathrm{F}$ & 6.41 & $4-5$ & 4 & $3-4$ & 4 & 4 & 4 & 4 & $4-5$ & $6-7$ \\
\hline II & S & 6.23 & $4-5$ & $4-5$ & 4 & 4 & 4 & 4 & 4 & $4-5$ & $6-7$ \\
\hline III & $\mathrm{F}$ & 8.63 & $4-5$ & 4 & $3-4$ & 4 & $3-4$ & 4 & 4 & 4 & $6-7$ \\
\hline III & $\mathrm{S}$ & 8.52 & $4-5$ & 4 & 4 & 4 & 4 & 4 & 4 & 4 & $6-7$ \\
\hline
\end{tabular}

I, II, and III are TGPLA synthetic thickeners prepared at 10, 50, and 98 \% of lactic acid, respectively. F: Freshly prepared, S: Stored for 3 dyes, St: Staining, Alt: Alteration.

Table S11. Comparison between K/S and fastness properties of wool fabric sample printed with prepared different TGPLA composite thickeners along with those printed using Sodium alginate when Basic used as a dye

\begin{tabular}{|c|c|c|c|c|c|c|c|c|c|c|c|}
\hline \multirow{4}{*}{ Thickening agent } & \multirow{4}{*}{ Storing Time } & \multirow{4}{*}{$\mathrm{K} / \mathrm{S}$} & \multicolumn{8}{|c|}{ Fasten properties } & \multirow{4}{*}{ Light } \\
\hline & & & \multirow{2}{*}{\multicolumn{2}{|c|}{ Rubbing }} & \multirow{2}{*}{\multicolumn{2}{|c|}{ Washing }} & \multicolumn{4}{|c|}{ Perspiration } & \\
\hline & & & & & & & \multicolumn{2}{|c|}{ Acidic } & \multicolumn{2}{|c|}{ Alkaline } & \\
\hline & & & Dry & Wet & St. & Alt & St. & Alt. & St. & Alt. & \\
\hline Sodium alginate & $\mathrm{F}$ & 17.21 & $4-5$ & $3-4$ & $3-4$ & 4 & $3-4$ & 4 & $3-4$ & 4 & $6-7$ \\
\hline Sodium alginate & S & 15.75 & $4-5$ & 4 & $3-4$ & 4 & $3-4$ & 4 & 4 & 4 & $6-7$ \\
\hline I & F & 19.85 & $4-5$ & 4 & $3-4$ & $3-4$ & 3 & 4 & $3-4$ & 4 & 6 \\
\hline I & S & 17.24 & $4-5$ & 4 & $3-4$ & $3-4$ & 3 & 4 & $3-4$ & 4 & 6 \\
\hline II & $\mathrm{F}$ & 17.39 & $4-5$ & 4 & $3-4$ & $3-4$ & 3 & 4 & 3 & 4 & 6 \\
\hline II & $\mathrm{S}$ & 11.34 & $4-5$ & 4 & 4 & 4 & 3 & 4 & 4 & 4 & 6 \\
\hline III & F & 19.77 & $4-5$ & 4 & $3-4$ & 4 & $3-4$ & 4 & 3 & 4 & $6-7$ \\
\hline III & $\mathrm{S}$ & 17.16 & $4-5$ & 4 & $3-4$ & $3-4$ & 3 & 3 & $3-4$ & 4 & $6-7$ \\
\hline
\end{tabular}

I, II, and III are TGPLA synthetic thickeners prepared at 10, 50, and 98 \% of lactic acid, respectively. F: Freshly prepared, S: Stored for 3 dyes, St: Staining, Alt: Alteration. 
Table S12. Comparison between K/S and fastness properties of cotton fabric sample printed with prepared different TGPLA composite thickeners along with those printed using commercial Synthetic thickener (Dico thick) when pigment used as a dye

\begin{tabular}{|c|c|c|c|c|c|c|c|c|c|c|c|}
\hline \multirow{4}{*}{ Thickening agent } & \multirow{4}{*}{ Storing Time } & \multirow{4}{*}{$\mathrm{K} / \mathrm{S}$} & \multicolumn{8}{|c|}{ Fasten properties } & \multirow{4}{*}{ Light } \\
\hline & & & \multirow{2}{*}{\multicolumn{2}{|c|}{ Rubbing }} & \multirow{2}{*}{\multicolumn{2}{|c|}{ Washing }} & \multicolumn{4}{|c|}{ Perspiration } & \\
\hline & & & & & & & \multicolumn{2}{|c|}{ Acidic } & \multicolumn{2}{|c|}{ Alkaline } & \\
\hline & & & Dry & Wet & St. & Alt & St. & Alt. & St. & Alt. & \\
\hline Dico thick & $\mathrm{F}$ & 10.95 & $3-4$ & 3 & 4 & 4 & $4-5$ & 4 & $4-5$ & 4 & 6 \\
\hline Dico thick & S & 12.13 & 3 & 3 & 4 & 4 & $4-5$ & 4 & $4-5$ & 4 & 6 \\
\hline I & $\mathrm{F}$ & 10.65 & $3-4$ & 3 & 4 & 4 & $4-5$ & 4 & $4-5$ & 4 & 6 \\
\hline I & S & 8.53 & $3-4$ & 3 & 4 & 4 & $4-5$ & 4 & $4-5$ & 4 & 6 \\
\hline II & $\mathrm{F}$ & 6.07 & $3-4$ & $3-4$ & 4 & 4 & 4-5 & 4 & $4-5$ & 4 & 6 \\
\hline II & S & 9.36 & 4 & 3 & 4 & 4 & $4-5$ & 4 & $4-5$ & 4 & 6 \\
\hline III & $\mathrm{F}$ & 10.32 & $3-4$ & 3 & 4 & 4 & $4-5$ & 4 & $4-5$ & 4 & 6 \\
\hline III & S & 9.06 & 3 & 3 & 4 & 4 & $4-5$ & 4 & $4-5$ & 4 & 6 \\
\hline
\end{tabular}

I, II, and III are TGPLA synthetic thickeners prepared at 10, 50, and $98 \%$ of lactic acid, respectively. F: Freshly prepared, S: Stored for 3 dyes, St: Staining, Alt: Alteration

Table S13. Comparison between K/S and fastness properties of polyester fabric sample printed with prepared different TGPLA composite thickeners along with those printed using commercial Synthetic thickener (Dico thick) when pigment used as a dye

\begin{tabular}{|c|c|c|c|c|c|c|c|c|c|c|c|}
\hline \multirow{4}{*}{ Thickening agent } & \multirow{4}{*}{ Storing Time } & \multirow{4}{*}{$\mathrm{K} / \mathrm{S}$} & \multicolumn{8}{|c|}{ Fasten properties } & \multirow{4}{*}{ Light } \\
\hline & & & \multirow{2}{*}{\multicolumn{2}{|c|}{ Rubbing }} & \multirow{2}{*}{\multicolumn{2}{|c|}{ Washing }} & \multicolumn{4}{|c|}{ Perspiration } & \\
\hline & & & & & & & \multicolumn{2}{|c|}{ Acidic } & \multicolumn{2}{|c|}{ Alkaline } & \\
\hline & & & Dry & Wet & St. & Alt & St. & Alt. & St. & Alt. & \\
\hline Dico thick & $\mathrm{F}$ & 9.19 & $3-4$ & 3 & $4-5$ & 4 & $4-5$ & 4 & $4-5$ & 4 & 6 \\
\hline Dico thick & S & 8.19 & 3 & 3 & $4-5$ & 4 & $4-5$ & 4 & $4-5$ & 4 & 6 \\
\hline I & $\mathrm{F}$ & 9.55 & $3-4$ & 3 & $4-5$ & 4 & $4-5$ & 4 & $4-5$ & 4 & 6 \\
\hline I & S & 10.17 & $3-4$ & $3-4$ & $4-5$ & 4 & $4-5$ & 4 & $4-5$ & 4 & 6 \\
\hline II & $\mathrm{F}$ & 8.75 & $3-4$ & 3 & $4-5$ & 4 & $4-5$ & 4 & $4-5$ & 4 & 6 \\
\hline II & S & 8.65 & $3-4$ & $3-4$ & $4-5$ & 4 & $4-5$ & 4 & $4-5$ & 4 & 6 \\
\hline III & $\mathrm{F}$ & 9.95 & $3-4$ & $3-4$ & $4-5$ & 4 & $4-5$ & 4 & $4-5$ & 4 & 6 \\
\hline III & $S$ & 8.69 & 3-4 & 3 & $4-5$ & 4 & $4-5$ & 4 & $4-5$ & 4 & 6 \\
\hline
\end{tabular}

I, II, and III are TGPLA synthetic thickeners prepared at 10, 50, and 98 \% of lactic acid, respectively. F: Freshly prepared, S: Stored for 3 dyes, St: Staining, Alt: Alteration

Table S14. Comparison between K/S and fastness properties of cotton/polyester fabric sample printed with prepared different TGPLA composite thickeners along with those printed using commercial Synthetic thickener (Dico thick) when pigment used as a dye

\begin{tabular}{|c|c|c|c|c|c|c|c|c|c|c|c|}
\hline \multirow{4}{*}{ Thickening agent } & \multirow{4}{*}{ Storing Time } & \multirow{4}{*}{$\mathrm{K} / \mathrm{S}$} & \multicolumn{8}{|c|}{ Fasten properties } & \multirow{4}{*}{ Light } \\
\hline & & & \multirow{2}{*}{\multicolumn{2}{|c|}{ Rubbing }} & \multirow{2}{*}{\multicolumn{2}{|c|}{ Washing }} & \multicolumn{4}{|c|}{ Perspiration } & \\
\hline & & & & & & & \multicolumn{2}{|c|}{ Acidic } & \multicolumn{2}{|c|}{ Alkaline } & \\
\hline & & & Dry & Wet & St. & Alt & St. & Alt. & St. & Alt. & \\
\hline Dico thick & $\mathrm{F}$ & 11.96 & $3-4$ & 3 & $4-5$ & 4 & $4-5$ & 4 & $4-5$ & 4 & 6 \\
\hline Dico thick & $\mathrm{S}$ & 12.93 & $3-4$ & $3-4$ & $4-5$ & 4 & $4-5$ & $4-5$ & $4-5$ & 4 & 6 \\
\hline I & $\mathrm{F}$ & 9.06 & $3-4$ & 3 & $4-5$ & 4 & $4-5$ & 4 & $4-5$ & 4 & 6 \\
\hline I & $\mathrm{S}$ & 12 & $3-4$ & 3 & $4-5$ & 4 & $4-5$ & 4 & $4-5$ & 4 & 6 \\
\hline II & $\mathrm{F}$ & 10.12 & 4 & 4 & $4-5$ & 4 & $4-5$ & 4 & $4-5$ & 4 & 6 \\
\hline II & S & 8.13 & $3-4$ & $3-4$ & $4-5$ & 4 & $4-5$ & 4 & $4-5$ & 4 & 6 \\
\hline III & $\mathrm{F}$ & 10.78 & $3-4$ & 3 & $4-5$ & 4 & $4-5$ & 4 & $4-5$ & 4 & 6 \\
\hline III & S & 8.8 & $3-4$ & $3-4$ & $4-5$ & 4 & $4-5$ & 4 & $4-5$ & 4 & 6 \\
\hline
\end{tabular}

I, II, and III are TGPLA synthetic thickeners prepared at 10, 50, and $98 \%$ of lactic acid, respectively. F: Freshly prepared, S: Stored for 3 dyes, St: Staining, Alt: Alteration 
Table S15. Comparison between K/S and fastness properties of acrylic fabric sample printed with prepared different TGPLA composite thickeners along with those printed using commercial Synthetic thickener (Dico thick) when pigment used as a dye.

\begin{tabular}{|c|c|c|c|c|c|c|c|c|c|c|c|}
\hline \multirow{4}{*}{ Thickening agent } & \multirow{4}{*}{ Storing Time } & \multirow{4}{*}{$\mathrm{K} / \mathrm{S}$} & \multicolumn{8}{|c|}{ Fasten properties } & \multirow{4}{*}{ Light } \\
\hline & & & \multirow{2}{*}{\multicolumn{2}{|c|}{ Rubbing }} & \multirow{2}{*}{\multicolumn{2}{|c|}{ Washing }} & \multicolumn{4}{|c|}{ Perspiration } & \\
\hline & & & & & & & \multicolumn{2}{|c|}{ Acidic } & \multicolumn{2}{|c|}{ Alkaline } & \\
\hline & & & Dry & Wet & St. & Alt & St. & Alt. & St. & Alt. & \\
\hline Dico thick & F & 12.07 & $3-4$ & 3 & $4-5$ & 4 & $4-5$ & 4 & $4-5$ & 4 & 6 \\
\hline Dico thick & S & 11.86 & 3 & 3 & $4-5$ & 4 & $4-5$ & 4 & $4-5$ & 4 & 6 \\
\hline I & $\mathrm{F}$ & 11.68 & $3-4$ & 3 & $4-5$ & 4 & $4-5$ & 4 & $4-5$ & 4 & 6 \\
\hline I & S & 11.95 & 3 & 3 & $4-5$ & 4 & $4-5$ & 4 & $4-5$ & 4 & 6 \\
\hline II & $\mathrm{F}$ & 7.48 & $3-4$ & 3 & $4-5$ & 4 & $4-5$ & 4 & $4-5$ & 4 & 6 \\
\hline II & S & 6.88 & 3 & 3 & $4-5$ & 4 & $4-5$ & 4 & $4-5$ & 4 & 6 \\
\hline III & $\mathrm{F}$ & 12.69 & 3 & 3 & $4-5$ & 4 & $4-5$ & 4 & $4-5$ & 4 & 6 \\
\hline III & S & 11.11 & 3 & 3 & $4-5$ & 4 & $4-5$ & 4 & $4-5$ & 4 & 6 \\
\hline
\end{tabular}

I, II, and III are TGPLA synthetic thickeners prepared at 10, 50, and $98 \%$ of lactic acid, respectively. F: Freshly prepared, S: Stored for 3 dyes, St: Staining, Alt: Alteration

Table S16. Comparison between K/S and fastness properties of polyester/wool fabric sample printed with prepared different TGPLA composite thickeners along with those printed using commercial Synthetic thickener (Dico thick) when pigment used as a dye

\begin{tabular}{|c|c|c|c|c|c|c|c|c|c|c|c|}
\hline \multirow{4}{*}{ Thickening agent } & \multirow{4}{*}{ Storing Time } & \multirow{4}{*}{$\mathrm{K} / \mathrm{S}$} & \multicolumn{8}{|c|}{ Fasten properties } & \multirow{4}{*}{ Light } \\
\hline & & & \multirow{2}{*}{\multicolumn{2}{|c|}{ Rubbing }} & \multirow{2}{*}{\multicolumn{2}{|c|}{ Washing }} & \multicolumn{4}{|c|}{ Perspiration } & \\
\hline & & & & & & & \multicolumn{2}{|c|}{ Acidic } & \multicolumn{2}{|c|}{ Alkaline } & \\
\hline & & & Dry & Wet & St. & Alt & St. & Alt. & St. & Alt. & \\
\hline Dico thick & $\mathrm{F}$ & 10.79 & $3-4$ & 3 & $4-5$ & 4 & $4-5$ & 4 & $4-5$ & 4 & 6 \\
\hline Dico thick & S & 10.42 & 3 & $2-3$ & $4-5$ & 4 & $4-5$ & 4 & $4-5$ & 4 & 6 \\
\hline I & F & 11.62 & $3-4$ & 3 & $4-5$ & 4 & $4-5$ & 4 & $4-5$ & 4 & 6 \\
\hline I & S & 7.34 & 3 & 3 & $4-5$ & 4 & $4-5$ & 4 & $4-5$ & 4 & 6 \\
\hline II & $\mathrm{F}$ & 6.91 & 4 & $3-4$ & $4-5$ & 4 & $4-5$ & 4 & $4-5$ & 4 & 6 \\
\hline II & S & 6.66 & 4 & 3 & $4-5$ & 4 & $4-5$ & 4 & $4-5$ & 4 & 6 \\
\hline III & $\mathrm{F}$ & 9.75 & $3-4$ & 3 & $4-5$ & 4 & $4-5$ & 4 & $4-5$ & 4 & 6 \\
\hline III & $S$ & 8.25 & 3 & $2-3$ & $4-5$ & 4 & $4-5$ & 4 & $4-5$ & 4 & 6 \\
\hline
\end{tabular}

I, II, and III are TGPLA synthetic thickeners prepared at 10, 50, and 98 \% of lactic acid, respectively. F: Freshly prepared, S: Stored for 3 dyes, St: Staining, Alt: Alteration

Table S17. Comparison between K/S and fastness properties of nylon fabric sample printed with prepared different TGPLA composite thickeners along with those printed using commercial Synthetic thickener (Dico thick) when pigment used as a dye.

\begin{tabular}{|c|c|c|c|c|c|c|c|c|c|c|c|}
\hline \multirow{4}{*}{ Thickening agent } & \multirow{4}{*}{ Storing Time } & \multirow{4}{*}{$\mathrm{K} / \mathrm{S}$} & \multicolumn{8}{|c|}{ Fasten properties } & \multirow{4}{*}{ Light } \\
\hline & & & \multirow{2}{*}{\multicolumn{2}{|c|}{ Rubbing }} & \multirow{2}{*}{\multicolumn{2}{|c|}{ Washing }} & \multicolumn{4}{|c|}{ Perspiration } & \\
\hline & & & & & & & \multicolumn{2}{|c|}{ Acidic } & \multicolumn{2}{|c|}{ Alkaline } & \\
\hline & & & Dry & Wet & St. & Alt & St. & Alt. & St. & Alt. & \\
\hline Dico thick & $\mathrm{F}$ & 6.77 & 4 & $2-3$ & $4-5$ & 4 & $4-5$ & 4 & $4-5$ & 4 & 6 \\
\hline Dico thick & S & 5.64 & $3-4$ & $3-4$ & $4-5$ & 4 & $4-5$ & 4 & $4-5$ & 4 & 6 \\
\hline I & $\mathrm{F}$ & 9.45 & 4 & 3 & $4-5$ & 4 & $4-5$ & 4 & $4-5$ & 4 & 6 \\
\hline I & S & 9.16 & 3 & 3 & $4-5$ & 4 & $4-5$ & 4 & $4-5$ & 4 & 6 \\
\hline II & $\mathrm{F}$ & 5.32 & 4 & 3 & $4-5$ & 4 & $4-5$ & 4 & $4-5$ & 4 & 6 \\
\hline II & S & 6.60 & 3 & 3 & $4-5$ & 4 & $4-5$ & 4 & $4-5$ & 4 & 6 \\
\hline III & $\mathrm{F}$ & 7.94 & 4 & 3 & $4-5$ & 4 & $4-5$ & 4 & $4-5$ & 4 & 6 \\
\hline III & S & 5.98 & 4 & 3 & $4-5$ & 4 & $4-5$ & 4 & $4-5$ & 4 & 6 \\
\hline
\end{tabular}

I, II, and III are TGPLA synthetic thickeners prepared at 10, 50, and $98 \%$ of lactic acid, respectively. F: Freshly prepared, S: Stored for 3 dyes, St: Staining, Alt: Alteration 


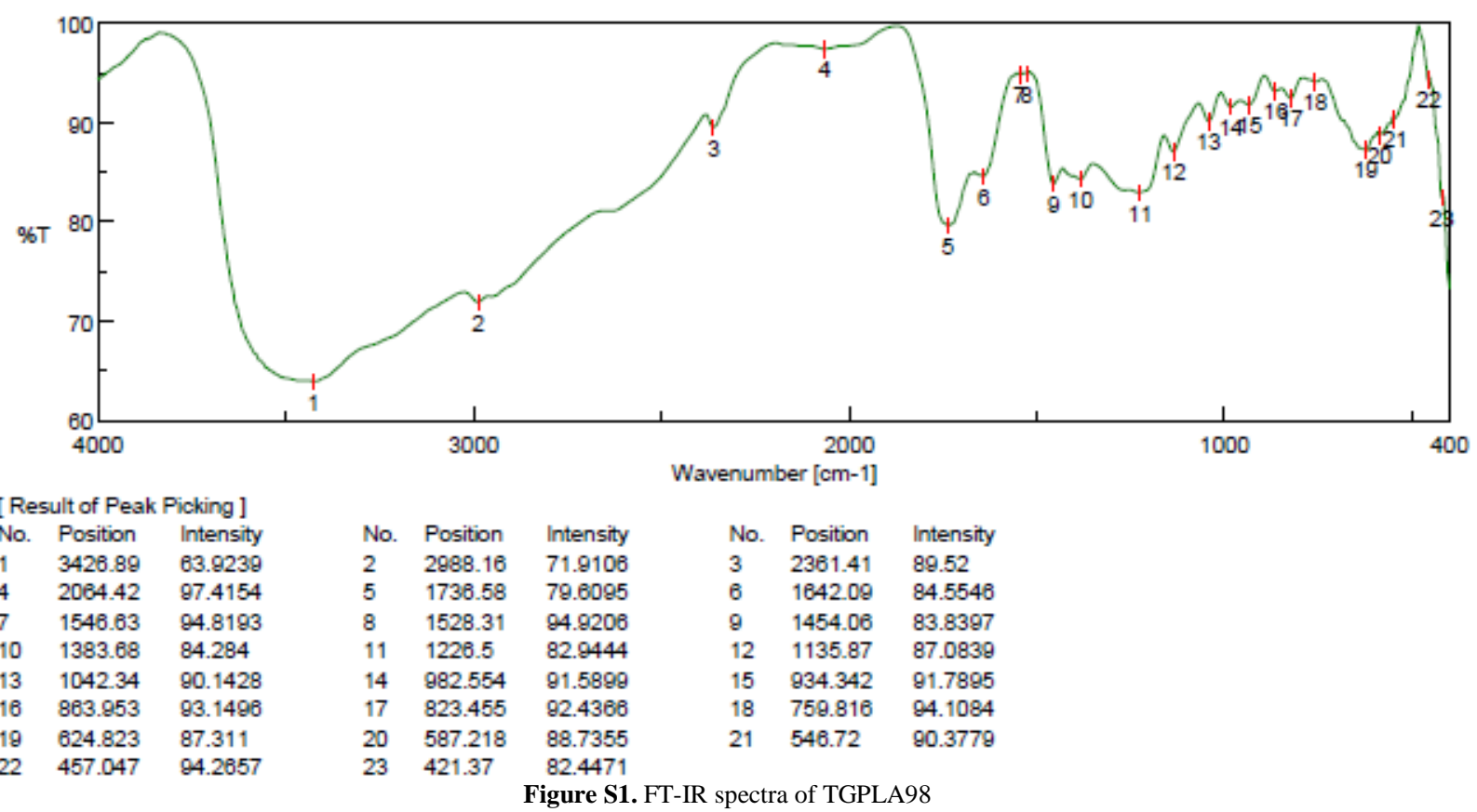

stustir-t-tans-nt

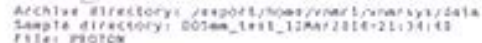

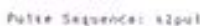

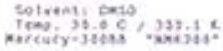

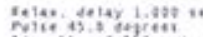

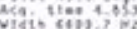

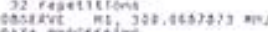

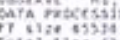

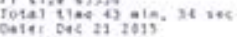

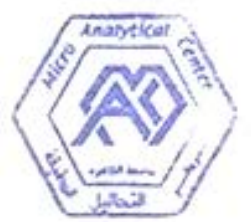

Figure S1. FT-IR spectra of TGPLA98

\section{0}

$\begin{array}{lllllllll}\text { No. } & \text { Position } & \text { Intensity } & \text { No. } & \text { Position } & \text { Intensity } & \text { No. } & \text { Position } & \text { Intensity } \\ 1 & 3426.89 & 63.9239 & 2 & 2988.16 & 71.9106 & 3 & 2361.41 & 89.52 \\ 4 & 2064.42 & 97.4154 & 5 & 1736.58 & 79.6095 & 6 & 1642.09 & 84.5546 \\ 7 & 1546.63 & 94.8193 & 8 & 1528.31 & 94.9206 & 9 & 1454.06 & 83.8397 \\ 10 & 1383.68 & 84.284 & 11 & 1226.5 & 82.9444 & 12 & 1135.87 & 87.0839 \\ 13 & 1042.34 & 90.1428 & 14 & 982.554 & 91.5899 & 15 & 934.342 & 91.7895 \\ 16 & 863.953 & 93.1496 & 17 & 823.455 & 92.4366 & 18 & 759.816 & 94.1084 \\ 19 & 624.823 & 87.311 & 20 & 587.218 & 88.7355 & 21 & 546.72 & 90.3779 \\ 22 & 457.047 & 94.2657 & 23 & 421.37 & 82.4471 & & & \\ & & & & & & \end{array}$

